\title{
Effect of speaking rate and contrastive stress on formant dynamics and vowel perception
}

\author{
Michel Pitermann \\ Department of Psychology, Queen's University \\ Kingston, Ontario, Canada KYL $3 N 6$ \\ mpiter@psyc.queensu.ca
}

(Abbreviated title: Formant dynamic and kinematic modeling

Received)

\begin{abstract}
Vowel formants play an important role in speech theories and applications, however, the same formant values measured for the steady-state part of a vowel can correspond to different vowel categories. Experimental evidence indicates that dynamic information can also contribute to vowel characterization. Hence dynamically modeling formant transitions may lead to quantitatively testable predictions in vowel categorization. Because the articulatory strategy used to manage different speaking rates and contrastive stress may depend on speaker and situation, the parameter values of a dynamic formant model may vary with speaking rate and stress. In most experiments speaking rate is rarely controlled, only 2 or 3 rates are tested, and most corpora contain just a few repetitions of each item. As a consequence, the dependence of dynamic models on those factors is difficult to gauge. This article presents a study of 2,300 [iai] or [i $\varepsilon$ i] stimuli produced by two speakers at 9 or 10 speaking rates in a carrier sentence for 2 contrastive stress patterns. The corpus was perceptually evaluated by naive listeners. Formant frequencies
\end{abstract}


were measured during the steady-state parts of the stimuli, and the formant transitions were dynamically and kinematically modeled. The results indicate that (1) the corpus was characterized by a contextual assimilation instead of a centralization effect; (2) dynamic or kinematic modeling was equivalent as far as the analysis of the model parameters was concerned; (3) the dependence of the model parameter estimates on speaking rate and stress suggests that the formant transitions were sharper for high speaking rate, but no consistent trend was found for contrastive stress; (4) the formant frequencies measured in the steady-state parts of the vowels were sufficient to explain the perceptual results while the dynamic parameters of the models were not.

PACS number: $43.70,43.71$ 


\section{INTRODUCTION}

Previous studies indicate that the first two formant frequencies measured in the steadystate part of a vowel plays an important role in its characterization (Helmholtz, 1885 1954; Joos, 1948; Delattre, Liberman, Cooper and Gerstman, 1952). However the formants of the same vowel uttered by different speakers, in different contexts, at different speaking rates and with different stress patterns exhibit a lot of variability (Joos, 1948; Peterson and Barney, 1952; Stevens and House, 1963; Lindblom, 1963).

The formant dependence on speaking rate and stress is usually described either by a centralization process or a context assimilation. Some studies have reported that formant frequencies of unstressed vowels or vowels uttered at fast speaking rates shift to a more central position around $(500,1500) \mathrm{Hz}$ in the $\left(F_{1}, F_{2}\right)$ plane (Joos, 1948; Koopmans-van Beinum, 1980). This is called centralization effect. A traditional view is that the vocal tract tends to become more similar to its "schwa" configuration for unstressed vowels and for fast utterances. In contrast, other studies have reported that vowel formants shift toward the values of their context instead of a central position (Stevens and House, 1963; Moon and Lindblom, 1994). This is called contextual assimilation.

An important issue related to vowel formant variability is that different vowels can be characterized by the same steady-state values of the first two formants (Joos, 1948; Peterson and Barney, 1952; Stevens and House, 1963). Because static formant values do not seem sufficient to identify vowels, dynamic information is also analyzed.

Lindblom and Studdert-Kennedy (1967) showed that vowels were categorized differently when they were presented in semi-vowel context instead of isolation. In context, listeners heard formant values extrapolated beyond the values actually reached at the end of the transitions. This perceptual overshoot was confirmed by Nearey (1989) for occlusive contexts. But another perceptual experiment presented in Nearey's article showed that the classification of sustained vowels was influenced by the presence of other sustained vowels. Hence an acoustical contrast between stationary segments may also contribute to a percep- 
tual overshoot. Some results conversely suggest that listeners can perceive formant values averaged over a full syllable, i.e. a value closer to the vowel context than the value actually reached (Pols and van Son, 1993). van Son (1993) proposed that a listener would exhibit a perceptual overshoot only when the vowel context has been identified.

Strange $(1989 a, b)$ presented several experiments where the vowel steady-state part of natural CVC stimuli were excised from the speech signals and replaced by silence. The perceptual scores of these silent-center stimuli were at least as good as the scores of the excised stationary segments presented in isolation. She proposed the theory of dynamic specification of vowels stating that vowels in continuous speech are better identified by coarticulation with their context than by information intrinsic to the vowel nuclei. Strange's results were replicated with adults and prelingual babies aged between 6 and 12 months for one German and one English vowel contrast (Polka and Bohn, 1996), and with adults for the 14 German monophtongs (Strange and Bohn, 1998). On the other hand Nearey and Assmann (1986) showed that isolated Canadian English monophtongs were slightly diphtongized, i.e. the steady-state stimuli contained significant formant frequency change over time. The same level of diphtongization was found for the vowels produced in context. The results of their experiments suggested that listeners may rely more on this vowel-inherent spectral change (VISC) than dynamic specification to identify vowels. Hence, vowels in continuous speech would be better characterized by their nuclei than by coarticulation with context. Andruski and Nearey (1992) showed perceptual results contradicting dynamic specification of vowels, but compatible with VISC. Jenkins, Strange and Trent (1999) presented an experiment based on the silent-center paradigm showing the opposite, i.e. results contradicting VISC theory but compatible with dynamic specification of vowels.

Automatic classification procedures also showed that dynamic information may help to categorize vowels. Cassidy and Harrington (1992) compared the performance of a neural network in vowel categorization for logatomes [CVd] using either static or dynamic information. The neural network used either formant transitions or stationary formant values duplicated several times in order to have the same segment durations in both cases. The network had 
higher identification scores when dynamic information was available. In Beautemps (1993) a neural network could categorize [iai] and [i i i] speech signals when only one 26-ms segment of each $F_{1}$ transition of [iV] stimuli was available. Conversely the network failed a complete categorization when the first three formants values measured in the stationary part were used. In contrast with the two previous works Watson and Harrington (1999) showed that a Gaussian classification algorithm could categorize vowels in a /h_d/ or / $\mathrm{h} /$ context only on the base of vowel duration plus formant values measured in the steady-state part of the vowel. But /h_d/ and /h_/ contexts produce less coarticulation than other consonant contexts (Stevens and House, 1963). The dynamics involved in the transitions could therefore be insufficient to play a role.

As Nearey (1989) proposed, all the previous contradicting results suggest that listeners may use many strategies together, but the perceptual weights given to of each of them would depend on the situation and task. Perceptual results and automatic classification procedures indicate that some of these strategies may rely on formant dynamics. Its characterization could thereby improve our understanding of vowel categorization.

There are no good theories predicting formant dynamics or what dynamic features could be used in vowel classification. It is not even clear whether the shape of a formant transition plays a perceptual role. Carré, Chennoukh, Lindblom and Divenyi (1994) activated the commands of a vocal tract model (Carré and Mrayati, 1992) with different mathematical functions (linear, parabola, sine, etc.) to synthesize [iai] stimuli. An analysis of the formant transitions in the $\left(F_{1}, F_{2}\right)$ plane showed that the shape of the transition varied with the mathematical form of the model commands. The perception of [a] was affected only when the canonical formant frequencies corresponding to a good [a] were far from being reached. Nábĕlek and Ovchinnikov (1997) perceptually compared linear and quadratic formant contours in a vowel identification task. The two formant contours led to the same categorization.

Other results suggest that the shape of formant transitions might change vowel categorization. Divenyi, Lindblom and Carré (1995) showed that perceptual overshoot depends 
on transition slope. In Carré, Chennoukh, Jospa and Maeda (1996), [abi] stimuli were synthesized by means of a vocal tract model (Carré and Mrayati, 1992) with different phasing between the consonant and vowel gestures. The shape of the formant transitions varied with this "articulatory" phasing. For some formant transitions, listeners heard an extra vowel. The perceptual results were [arbi], [abi] or [abei] depending on the "articulatory" phasing. As a consequence, even if a description of formant dynamics may not need to be highly accurate in all situations (Carré et al., 1994; Nábělek and Ovchinnikov, 1997), its characterization may lead to testable predictions in vowel identification.

Some attempts have been done to describe formant dynamics. For example, Broad and Clermont (1987) offered several models of formant transition. The more elaborate one was an exponential fit and it had good variance explanation. In van Son and Pols (1992) a professional radio speaker read a Dutch text at normal then fast speaking rate. Vowel duration was normalized during formant extraction, i.e. all formant time series segments corresponding to vowels had the same length. No differences were found between the formant contours when the two speaking rates were compared, but this representation helped to distinguished front from back vowels. The normalized time series were also fitted to Legendre polynomial expansions in order to characterize the shape of the formant transitions, and the same results were found, i.e. no fit differences for the two speaking rates and the same classification performance. In Watson and Harrington (1999), formant time series were fitted to discrete cosine expansions to characterize formant contours of vowels uttered in [h_d] and [h_] contexts. This representation helped to identify the diphthongs. However good, none of these formant contour characterizations were proven sufficient to identify all vowels in a general context. New attempts may therefore be needed. This work presents models of formant dynamics and kinematics and analyzes how their parameters and the formant frequencies depended on speaking rate and contrastive stress.

To model the dynamics of the first two formants, the system underlying their production was assumed stationary during an analyzed transition. $F_{1}$ and $F_{2}$ were modeled separately for two reasons. Firstly, it can be seen in Pitermann (1996) that the first two formants of the 
corpus presented here ([iai] and [i $\varepsilon \mathrm{i}]$ stimuli) were not synchronized. Hence, two independent models appeared a better choice. Secondly, there are more mathematical tools available to guide one-dimensional than two-dimensional modeling.

It is often presumed that an articulatory movement can approximately be described by a second-order linear model (Sonoda, 1987; Laboissière, Ostry and Perrier, 1995). A corpus with simple articulatory movements was chosen (only a front-back-front tongue movement and a jaw opening-closing may be involved in the production of [iai] and [iei] stimuli). As a consequence, and because it is the simplest model, the linear model was firstly selected.

In a revision of Lindblom's original target undershoot model (Lindblom, 1963), Moon and Lindblom (1994) proposed to describe the relationship between vowel duration and steadystate formant frequency by a second-order linear model at critical damping. Although the mathematical forms of a formant time series and of the relationship between vowel duration and formant frequency may be different, these two concepts may be closely tied. This model was therefore chosen too. One may object that the second-order linear model at critical damping is a special case of the linear model firstly selected, hence a study of the latter should be sufficient to back up or contradict the former. This point will be discussed in the "Results" section II D.

A small-order linear model cannot accurately describe an asymmetrical transition. The first- and second-half of each formant transition were therefore separately modeled. To model a whole transition, a nonlinear dynamic model having the logistic curve among its solutions was implemented. It will be called the logistic model in this article.

Fitting the parameters of a dynamic model or of one of its solutions (a kinematic model, i.e. a curve fitting) may lead to different results. The reason is that the kinematic and dynamic model equations are different, and the associated cost functions to be minimized are different too. For example, an exponential is the solution of a first-order linear dynamic model. Fitting an exponential or a linear model may be different. A linear least-squares method can be used for the linear model, not for the exponential. Hence, the two fits may lead to different results. 
For each dynamic model, one of its solutions was therefore tested: an exponential, a combination of an exponential and time, and a logistic curve respectively for the linear, second-order linear at critical damping, and logistic models. Only the exponential was chosen as the solution for the linear model because, as will be justified in the "Results" section II D, the first order was sufficient to describe the half-transitions of the corpus.

The model equations are summarized in Table I and their graphics can be seen in Figure 1. Three general criticisms can be made about most studies dealing with speaking rate and formants extracted from natural speech. Firstly, many corpora include a lot of different phonemes in different contexts with few examples of each particular case, e.g. (Kuwabara, 1985; van Son and Pols, 1992). Many sources of variability may thereby be responsible for the results, and it is difficult to assess the role of each of them. Secondly, only two or three speaking rates are usually used in those experiments, e.g. (Imaizumi and Kiritani, 1989; Loevenbruck and Perrier, 1993). Thirdly, the speaking rates are generally not controlled. The speakers are usually asked to speak slowly, naturally or fast, e.g. (van Son and Pols, 1992; Beautemps, 1993).

In contrast, this article presents an analysis of approximately 2,300 [iai] or [i $\varepsilon \mathrm{i}$ ] stimuli uttered by two speakers in a carrier sentence at 9 or 10 speaking rates controlled by metronome for two contrastive stress patterns. The stimuli were categorized during a perceptual task. The first two formants were measured in the stationary parts of the vowels, and three dynamic and three kinematic models were fitted to the formant transitions. The contribution of static and dynamic information to the perceptual results was assessed, and the centralization versus context assimilation issue was discussed in the light of the results. The dynamic and kinematic models gave a quantitative description of the formant contours to be related to the perceptual results. The high number of speaking rates controlled by metronome and the high number of repetitions of each corpus item provided more accuracy for the relationship between speaking rate and variables than what is usually presented. 


\section{METHOD}

\section{A. Speech material}

The first two formants of [ia] and [i $\varepsilon]$ transitions taken from [iVi] segments were analyzed (V was $[\mathrm{a}]$ or $[\varepsilon]$ ). The segments were excised from two carrier sentence portions [il $i$ Vimedia] taken from two French sentences: "Il y a immédiatement souscrit" ("He immediately subscribed to it") and "Il y est immédiatement retourné" ("He immediately returned to it"). The carrier sentence portions were produced in an anechoic room by two French speaking male speakers, $\mathrm{A}$ and $\mathrm{B}$, at 10 and 9 different speaking rates respectively. All speaking rates were controlled by metronome. The speakers had to synchronize the beginning of the carrier segment with a metronome beat, and the plosion of $[\mathrm{d}]$ with the following one. The metronome rhythms were 48, 54, 60, 69, 80, 88, 96, 104, 112 and 120 beats/minute. The lowest rate was very slow for the speakers, the highest was the limit bearable by each of them, i.e. the highest rate before they could not synchronize their production and the metronome beats. The speakers were asked to produce the whole corpus placing a contrastive stress on $\mathrm{V}$, then a second time without any contrastive stress. I controlled the recording sessions, and each combination of speaking rate, contrastive stress and vowel was repeated until 30 satisfying examples were produced. Around 4,600 formant transitions were therefore analyzed $(2$ vowels $\times 2$ contrastive stresses $\times 19$ speaking rates $\times 30$ repetitions $\times 2$ formants $)$.

The signals were recorded with a microphone (NEUMAN U $87 \mathrm{P} 48$ ) connected to a 8-kHz low-pass filter and sampled at $32 \mathrm{kHz}$ by a DAT recorder (SONY PCM-2300, 16bit recording). The cutoff frequency of the low-pass filter was 8,000 Hz, and its slope was $360 \mathrm{~dB} /$ octave. The corpus was then digitally transfered from the DAT to a computer. The sampling frequency was thereafter reduced to $16 \mathrm{kHz}$ on the computer by taking away one sample out of two. The 8-kHz low-pass filter used during the recording sessions avoided any potential aliasing problems when the sampling frequency was reduced from 32 to $16 \mathrm{kHz}$. 


\section{B. Perceptual analysis}

The corpus was perceptually evaluated to spot improperly produced data, i.e. [iVi] speech signals for which the perceptual identity of $\mathrm{V}$ did not correspond to the instructions given to the speakers.

Eight French speaking participants between 22 and 30 years of age and without phonetic training took part in the experiment. No listeners reported suffering from impaired hearing. The data of the last listener was lost due to an experimental mistake.

For each listener, one list per talker was made up of 4 different [iVi] segments for each combination of rate, stress and vowel. For example, one list corresponding to talker B contained 144 items $(4$ occurrences $\times 9$ rates $\times 2$ stresses $\times 2$ vowels $)$. Since the corpus was large enough, no speech signals were used twice. The stationary parts of [i] were not kept, thus the stimuli sounded more $[\mathrm{jVj}]$ than $[\mathrm{iVi}]$. To eliminate onset and offset noise, the signal amplitude was multiplied by a linear 12.5-ms slope at the beginning and end of each stimulus.

Each subject listened to one list per speaker. The stimuli were randomly presented to each listener. Two successive items were separated by a 3-second silence during presentation. Four participants started with a speaker-A list, the other three with a speaker-B. Before starting the perceptual test, the subjects heard 20 samples from another list to adjust the signal intensity at a comfortable level and become familiar with the assignment.

The task was to write down each vowel identified between the two [i]. When a listener hesitated, he or she was allowed to leave a blank or choose the closest vowel. No information was given about the carrier sentence, the vowel to be identified or the phonetic transcription to use. It was therefore a test with open answers.

The signals were played back on BEYER Beyerdynamic dt325 headphones connected to a Sparc 20 SUN workstation containing a 16-bit linear digital to analog converter.

Owing to the loss of data, the results of only 28 out of 30 repetitions of each corpus item were available. However, this only amounted to a loss of less than $7 \%$ of the perceptual 
data.

\section{Formant measurements}

Formant frequency estimation is a mathematically ill-formed problem, i.e. there are no theorems proving that a solution exists, that a solution is unique and that a solution continuously depends on the initial conditions. Hence, several heuristics may be tried for each problem. In this study, the outcome of dynamically modeling a formant transition may be influenced by the quality of the formant extractor used. Several formant estimate procedures were therefore compared: a pure LPC method (Atal and Hanauer, 1971), a method combining the results of one cepstral and two LPC algorithms (Soquet, 1995a), and a pitchsynchronous method (Soquet, 1995b). The pitch-synchronous method produced cleaner formant time series than the two other algorithms, but all techniques led to the same conclusion as far as dynamic modeling was concerned. I thereby kept the LPC-based formant extractor to stick to the simplest tool.

The speech signals were sampled at $16 \mathrm{kHz}$, then modified by means of a $(1-0.95 z)$ preemphasis filter (in $z$-transform notation). Every $9 \mathrm{~ms}$, the first two formant frequencies were estimated by means of an 18 order covariance LPC (Linear Predictive Coding) analysis in a 26-ms rectangular window. The lowest pole frequency was assigned to the first formant, the second pole frequency to the second formant.

All formant time series were visually examined to check for formant misclassifications. Formant insertions were manually erased. When a formant omission was found, the time series of the first two formants of the speech signal were reestimated by means of a LPC model with a different order. If no orders in the $[10,20]$ interval could produce two time series without omissions, the speech signal was discarded. Less than $1 \%$ of the corpus was rejected this way.

Values $0.70,0.75,0.80,0.85,0.90$ and 0.95 were tested for the preemphasis filter. Since these values did not lead to different results as far as dynamic modeling of formant transitions 
was concerned, 0.95 was selected in order to be consistent with the literature (Makhoul, 1975).

For each corpus item, formant frequencies of vowel $[\mathrm{V}]$ were defined as the $F_{1}$ maximum and $F_{2}$ minimum of their time series. This means that a formant frequency was measured at the time coordinate for which the time series took on a nearly horizontal slope inside segment $[\mathrm{V}]$.

\section{Formant modeling}

\section{Choice of models}

Three dynamic models and one solution of each model was fitted to each formant transition. The three dynamic models were a linear, a second-order linear at critical damping and a logistic model. The three kinematic models were an exponential, a combination of exponential and time, and a logistic curve. The model equations are summarized in Table I and their graphics can be seen in Figure 1.

\section{Parameters estimations}

The coefficients of the six models were estimated by means of least-squares methods for each formant transition. Because there were approximately 4,600 formant transitions in the corpus, around 4,600 sets of parameters were estimated for each model. The merit function was the classical sum of the squared differences between model-generated and measured values. The coefficients of the linear model (1) that gave rise to the minimum merit function were determined by means of a conventional linear method. The coefficients of the

nonlinear difference equation (5) and curves (2), (4) and (6) were estimated by means of the Levenberg-Marquardt algorithm. This is an optimizer that gradually switches from a steepest descent method to a quadratic approximation when the merit function approaches a minimum (Press, Teukolsky, Vetterling and Flannery, 1992). 
The merit function of the second-order linear model at critical damping (3) was

$$
E=\frac{1}{N-2} \sum_{i=3}^{N}\left(x_{i}-a_{0}-a_{1} x_{i-1}+\frac{a_{1}^{2}}{4} x_{i-2}\right)^{2}
$$

where $E$ is the merit function, $x_{i}$ is the $i$ th sample of the time series, $N$ is the length of the time series, and $a_{0}$ and $a_{1}$ are the parameters of the model. Setting to 0 the first derivative of the merit function with respect ot $a_{0}$ and $a_{1}$ leads to a nonlinear system of two equations:

$$
\left\{\begin{aligned}
a_{0} & =\bar{x}_{n}-a_{1} \bar{x}_{n-1}+\frac{a_{1}^{2}}{4} \bar{x}_{n-2} \\
0 & =\sigma_{x_{n-2}}^{2}\left(\frac{a_{1}}{2}\right)^{3}-3 \sigma_{x_{n-1} x_{n-2}}\left(\frac{a_{1}}{2}\right)^{2}+\left(2 \sigma_{x_{n-1}}^{2}+\sigma_{x_{n} x_{n-2}}\right)\left(\frac{a_{1}}{2}\right)-\sigma_{x_{n} x_{n-1}}
\end{aligned}\right.
$$

where:

$$
\begin{aligned}
\bar{x}_{n-k} & =\frac{1}{N-2} \sum_{i=3}^{N} x_{i-k} \\
\sigma_{x_{n-k}}^{2} & =\frac{1}{N-2} \sum_{i=3}^{N}\left(x_{i-k}-\bar{x}_{n-k}\right)^{2} \\
\sigma_{x_{n-k} x_{n-l}} & =\frac{1}{N-2} \sum_{i=3}^{N}\left(x_{i-k}-\bar{x}_{n-k}\right)\left(x_{i-l}-\bar{x}_{n-l}\right) .
\end{aligned}
$$

The procedure to estimate $a_{0}$ and $a_{1}$ for a time series $x_{i}$ was straightforward. The thirddegree polynomial (8) was solved to produce 1 or 3 real roots. For each $a_{1}$ (1 or 3 values), the corresponding $a_{0}$ value was calculated by means of the first equation of (8). The couple $\left(a_{0}, a_{1}\right)$ that gave rise to the minimum merit function $(7)$ was selected.

The order of the linear difference equation (1) was determined by analyzing the random variable $\left(\hat{a}_{i}-a_{i}\right) / \hat{\sigma}\left(\hat{a}_{i}\right)$, where $a_{i}$ is the $i$ th unknown coefficient of the model for the full population of all possible formant transitions, $\hat{a}_{i}$ is its estimate based on a sample of the population, and $\hat{\sigma}\left(\hat{a}_{i}\right)$ is the estimate of its standard error. When the conditions of application of a linear regression are met, this variable is characterized by a $t$-distribution (Spiegel, 1988, chapter 14 , pp. 300). The null hypothesis " $H_{0}: a_{i}=0$ " could thereby be tested at 0.05 level for any coefficient of the model. The model order was iteratively increased by 1 until the null hypothesis for the highest-order coefficient $a_{k}$ could not be rejected. The last but one order was selected. 
The [iVi] segmentation procedures were the following. Complete formant transitions between preceding [i] and [V] were modeled by means of the logistic models (5) and (6). The first sample of a transition was manually chosen near the end of the horizontal part of the formant time series of the [i], the last sample was the next maximum for $F_{1}$ or minimum for $F_{2}$.

In contrast to models (5) and (6), only the half-transitions before or after the points of inflection were modeled by means of the four other models. The position of each point of inflection was determined by means of parameter $t_{0}$ of the logistic curve (6). To increase $t_{0}$-estimate accuracy, a speaker was assumed to use the same articulatory gesture for the 30 repetitions of a corpus item. Accordingly, the 30 transitions were presumed to be characterized by a single coefficient vector $(a, b, c)$ while each transition assumed a different time position $t_{0}$. I used the Viterbi algorithm in order to estimate a unique $(a, b, c)$ vector and 30 different $t_{0}$ values simultaneously using the 30 transitions (Viterbi, 1967). The method consisted of alternatively estimating either one structural vector $(a, b, c)$ or the 30 time positions $t_{0}$ while keeping the other coefficient class fixed until convergence was achieved.

\section{RESULTS}

\section{A. Transition length and speaking rate}

Figure 2 shows how the duration of the $F_{1}$ transitions varied with speaking rate. Each point of the plot is a median of 30 durations computed from 30 repetitions of a corpus item in one speaking rate and contrastive stress condition. The same pattern was observed for $F_{2}$.

The graphics show that formant transition duration decreased as speaking rate increased. A floor effect appeared after 80 beats/minute, although the speakers continued to increase their speaking rate. Other parts of the segment sentence were therefore shortened for the highest speaking rates. 


\section{B. Perceptual evaluation of the corpus}

The purpose of the perceptual analysis was to spot the [iVi] tokens that were incorrectly produced.

Around $99.5 \%$ of vowels $\mathrm{V}$ were labeled /a/, / $/ \varepsilon /$ or $/ \mathrm{e} /$. Because $[\mathrm{e}]-[\varepsilon]$ distinction depended on regional accent, and because the listeners did not receive any references, labels /e/ were replaced by $/ \varepsilon /$. This way, $99.5 \%$ of the labels became /a/ or $/ \varepsilon /$, and most

mistakes were considered to be confusions between $[a]$ and $[\varepsilon]$.

More than $90 \%$ of vowels $[\varepsilon]$ were correctly identified for 35 speaking rate and contrastive stress groups out of $38(38=9$ rates $\times 2$ stresses +10 rates $\times 2$ stresses $)$. The identification scores of the remaining 3 conditions were $86 \%$.

Figure 3 shows the average identification scores normalized by the total number of answers of the seven listeners for vowel [a] as a function of speaking rate.

More than $90 \%$ of speaker A's [a] were correctly identified for the seven lowest speaking rates. The identification scores were below $85 \%$ for the other speaking rates.

The identification scores of speaker B's [a] were above $90 \%$ for only 9 categories out of 18 (the stressed [a] produced at the six lowest speaking rates and the unstressed [a] produced at the three lowest ones). For the other categories, the identification scores varied between 18 and $68 \%$.

\section{Formant measurements}

Processing about 4,600 formant transitions by means of six dynamic or kinematic models led to around 27,600 fits. Due to noise, some of them were physically impossible and should be considered as aberrations. To lower their impact on the statistical description of the corpus, the median and a similar measure to semi-interquartile range were chosen instead of the mean and square deviation. For consistency, the formants were described in the same way. 
Instead of the semi-interquartile range, the semi-68-\% range leaving $16 \%$ instead of $25 \%$ of the elements in each tail of the distribution was used. The advantage of the semi68-\% compared to the semi-interquartile range is that it has the same value as the standard deviation when the distribution is Gaussian. It is more convenient for comparisons when different statistical feature sets are used to describe the data.

Table II shows the medians and semi-68-\% ranges of the first two formants of [i], $[\varepsilon]$ and [a] for both speakers. Those values were computed across all repetitions, speaking rates and stresses. The variability of [i]'s formant frequencies was lower than [a]'s but similar to $[\varepsilon]$ 's.

Figure 4 shows the medians of the first two formants of each speakers' $[\varepsilon]$ and [a] computed for each set of 30 repetitions of a corpus item for all combinations of speaking rate and contrastive stress. The data is plotted as functions of speaking rate measured in metronome rhythm. Table III shows the results of two-factor (rate and stress) analyses of variance for all combinations of speaker, vowel and formant. All analyses of variance were carried out with a linear additive model with replication. Interactions between factors were included in the model.

\section{Model parameters}

The first step to analyze the corpus using a linear model (1) was to estimate its order $k$. To validate the first order, the null hypothesis that $a_{1}$ was equal to 0 was tested for each formant half-transition. The hypothesis was rejected at 0.05 level in $97.7 \%$ of the cases. Hence $a_{1}$ should be considered as different from 0 , so the first order was mandatory. For the second order, the null hypothesis was tested for $a_{2}$, and it was rejected at 0.05 level in $4.5 \%$ of the cases. Thus $a_{2}$ could not be considered as different from 0 , and the first order was sufficient to model the formant half-transitions. Accordingly, we used a first-order linear model and one of its solutions, an exponential.

Because a first-order linear model was sufficient to describe the data, the second-order model at critical damping proposed in Moon and Lindblom (1994) may appear inadequate. 
Being able to reject higher-order linear models, however, does not imply that a first-order linear model was underlying the data. It only means that, with the number of points available for each fit under the noise condition, no information could be found about higherorder parameters by a classical linear regression.

When a constraint between model parameters is added, the parameter space suitable for a fit is reduced. For example, when a linear second-order model at critical damping is responsible for some data, the noise can hide information about the second order. If the topology of the cost function is complex with many local minima, the fitting procedure may be easily caught in a local minimum far away from the global one. However, adding the critical constraint to the fitting process reduces the search in a 3 -D space $\left(a_{0}, a_{1}\right.$ and $a_{2}$ from equation (1) of Table I) to a search in a 2-D space $\left(a_{0}\right.$ and $a_{1}$ from equation (3)), i.e. in a plane. Hence the algorithm may converge to a better local minimum or the global one because the search is better guided by the fitting procedure. For this reason, a secondorder linear model at critical damping was also fitted to the transitions to follow Moon and Lindblom (1994) suggestion.

In the two selected linear models (1) and (3), $a_{1}$ indicated how fast a transition left an asymptote or came close to it and was dampened near it. The lower $a_{1}$, the faster a formant left or approached its asymptotic value and was dampened. It may therefore be expected that fast transitions were modeled with a low $a_{1}$ value.

Figures 5 and 6 show the medians of the $a_{1}$ estimates of the first-order linear model for each set of 30 repetitions of a corpus item for each combination of speaker, vowel, formant, contrastive stress and speaking rate. The data is presented as functions of speaking rate measured in metronome rhythm. Tables IV and V show the results of two-factor (stress and rate) analyses of variance for all combinations of speaker, vowel and formant. Figure 5 and Table IV refer to the first-half transitions, Figure 6 and Table V to the second-half.

The results produced by means of the two linear models (1) and (3) were slightly different in their details, but the conclusions of all analyses were the same. Therefore, only the firstorder linear model will be discussed in the rest of the paper. 
The exponential (2) and the kinematic model (4) were also fitted to the formant halftransitions. Since the parameters $a_{1}$ of the dynamic linear models were linked to their kinematic equivalents by $a_{1}=\exp (-q \Delta t)$, this value was computed for the kinematic models in order to compare the dynamic and kinematic results. Because the results were virtually identical and the kinematic models did not add any new information, those kinematic models will not be considered further in this paper.

Up to this point, only half-transition modeling has been presented because low-order linear models with constant parameters could not describe a full transition. The logistic models (5) and (6) permitted complete transition modeling.

In the logistic models, $d$ of (5) and $b$ of (6) played a similar role than $a_{1}$ and $q$ of the linear (1) and exponential (2) models, and the relation $d=\exp (-b \Delta t)$ was valid. In other words, $d$ or $b$ controlled the models when $t \rightarrow \pm \infty$, i.e. they described how a formant left its first asymptote and how it was dampened near the second one.

Figure 7 shows the medians of the $d$ values of each set of 30 repetitions of a corpus item for each combination of speaker, vowel, formant, contrastive stress and speaking rate. The data is plotted as functions of speaking rate measured in metronome rhythm. A smaller $d$ value means that the formant left the initial value and was dampened near the final one more abruptly. Table VI shows the results of two-factor (rate and stress) analyses of variance for all combinations of speaker, vowel and formant.

Once again, dynamic or kinematic modeling produced approximately the same results. The kinematic ones were therefore not included in this paper.

Because a second-half transition may be more influenced by following gestures than the first-half, the logistic models was also fitted to the first-half transitions. Modeling a first-half or a full transition led to similar results, except that there was more noise in the fits with the first-half. However, this increase in noise was expected because fewer points were included in the fits. The tables are not presented because they did not add any new information. The analysis simply indicated that the first- and second-half transitions were not different enough to lead to different conclusions. 
Table VII shows standard linear Pearson correlations measured between the perceptual scores and the median of the formant measurements or the medians of the model parameters.

Figure 8 plots in the $\left(F_{1}, F_{2}\right)$ plane the formant frequencies measured in the steady-state parts of the $[a]$ and $[\varepsilon]$ stimuli correctly identified in the perceptual test. Figure 9 shows a similar plot for the dynamic parameter $a_{1}$ of the linear model (1) fitted to the second-half transitions of $F_{1}$ or $F_{2}$ respectively for the $x$ or $y$ axis. The two ellipses formed by each vowel in the $\left(F_{1}, F_{2}\right)$ space are basically disjoint while the two vowels completely overlap in the dynamic parameter space. The same overlap was found for the parameter $a_{1}$ fitted to the first-half formant transitions and for the parameter $d$ of the nonlinear model (5) fitted to complete formant transitions.

\section{DISCUSSION}

As explained in the "Introduction" section, fitting a dynamic or an equivalent kinematic model can lead to different results. Both fits were compared for each model, and both fits produced similar results every-time. This simply means that the minimization processes of the merit functions of each dynamic and corresponding kinematic models found a minimum in the same area of their parameter spaces. Hence both kinds of models were equivalent as far as the analysis of their fitted parameters was concerned.

Figure 4 shows that the first two formants frequencies measured at the slowest speaking rate were close to $(400,1800) \mathrm{Hz}$ for both speakers' $[\varepsilon]$ and $(600-700,1400-1500) \mathrm{Hz}$ for speakers' [a]. Table II show that the first two formant frequencies of the context were close to $(250,1950) \mathrm{Hz}$ with small variation around those values. A contextual assimilation should therefore decrease $[\mathrm{a}]$ and $[\varepsilon] F_{1}$, and increase their $F_{2}$ for high speaking rates. In contrast, a centralization effect should not modify the [a] formant values much, but should increase [i] and $[\varepsilon] F_{1}$, and decrease their $F_{2}$. Figure 4 shows a contextual assimilation for both speakers because $[\varepsilon]$ and $[\mathrm{a}]$ formant frequencies were shifted away from a central position

toward [i] formants values for high speaking rates. Table III shows that the dependence of 
the formants on speaking rate and contrastive stress was statistically significant at 0.05 level in all cases.

This conclusion should not be generalized to any phoneme and context. The corpus was purposely chosen to make $[\mathrm{i}]$ stable and $[\varepsilon]$ and $[\mathrm{a}]$ varying. Firstly, French $[\mathrm{i}]$ is known to need more articulatory accuracy and to have more stable formants than other vowels (Majid, Abry, Boe and Perrier, 1987). Secondly, the carrier sentence [iliVimedia] was built to favor the context instead of $\mathrm{V}$.

The speakers did not manage their contrastive stress in the same way. Firstly Figure 4 shows that speaker A's formants were less influenced by contrastive stress than speaker B's, specially for [a] (each pair of solid or dotted line was closer for speaker A than B). Secondly speaker A's production seemed more regular than speaker B's. This can be seen on Figure 4 because speaker B's unstressed [a] uttered at the six highest speaking rates and those uttered at the three lowest ones seem different. The first two formants of the former were similar to the speaker's $[\varepsilon]$ formants while the formants of the latter were clearly distinct. The perceptual results support this hypothesis because the unstressed [a] identification scores were less than $50 \%$ correct for the six highest speaking rates while they were equal to $100 \%$ in the three other cases. On the other hand speaker B's stressed [a] formants were shifted more gradually toward the contextual formant values when speaking rate was increased. This pattern suggests that speaker B did not produce his stressed and unstressed [a] in the same way, like the other speaker did.

It is common to find data in the literature showing that speakers can increase, keep constant or decrease their articulatory strength when they stress a syllable or speak faster (Gay, Ushijima, Hirose and Cooper, 1974; Kuehn and Moll, 1976; Sonoda, 1987; Guenther, 1995; Marchal and Meynadier, 1996). Figure 6 suggests that the parameter $a_{1}$ of the linear model (1) fitted to the second-half transitions tended to be smaller for high speaking rates but did not exhibit any consistent trend for contrastive stress. This result means that formants approached their $F_{1}$ maximum or $F_{2}$ minimum faster for high speaking rates. Even if the trend was consistent, table $\mathrm{V}$ shows that it was seldom significant at 0.05 level. It could 
mean that the trend was partly masked by the noise and that processing a bigger corpus would confirm the results. Figure 5 shows that the results regarding the first-half transitions were less regular.

Figure 7 shows that modeling whole transitions by means of the logistic model (5) did not change the previous trend, although this trend appeared less clearly. The parameter $d$ was smaller for high speaking rates and the results were less clear for stress. This means that the formants tended to leave their initial and reached their final values more abruptly for high speaking rates. Once again, the trend was not always significant but consistent. The trend suggests that the speakers used more force in their articulatory gestures for high speaking rates. But the nonlinear relationships between the articulatory and acoustical levels may be misleading and this conclusion must be confirmed by articulatory or EMG data.

Experiments showed that vowels can be better categorized by dynamic than static information in some situations (Strange, 1989b; Strange, 1989a; Cassidy and Harrington, 1992; Beautemps, 1993; Jenkins et al., 1999). Table VII shows that the absolute values of the linear correlations between the perceptual scores and the medians of speakers' formants measured in the vowel steady-state parts were always greater than 0.8 for [a]. Because the perceptual scores of $[\varepsilon]$ were always close to $100 \%$, the correlations between those scores and the formant values are not informative. Only the [a] scores will be discussed.

It is interesting to find such a high correlation between the perceptual scores and the formant measurements for [a] because those measurements were made in the steady-state part of the vowels. It suggests that static information was more important than expected. To investigate this issue, the formant measurements of the 1,900 vowels [a] and [ $\varepsilon$ ] correctly identified in the perceptual experiment were plotted in the $\left(F_{1}, F_{2}\right)$ plane in Figure 8 . In this acoustical representation, all but 40 vowels [a] and [ $\varepsilon$ ] out of 1,900 formed two ellipses without intersection, i.e. [a] and [ $\varepsilon$ ] could be classified in this representation.

This result contrasts with Beautemps (1993) results showing greater overlap between [a] and $[\varepsilon]$ of a smaller but similar corpus. However he only used 40 stimuli produced by one speaker so his results should not be generalized. 
On the other hand my results corroborate one Peterson and Barney (1952) finding. They showed many measurements of the first two formants of 10 English vowels in the $\left(F_{1}, F_{2}\right)$ plane, and the vowels overlapped in this space. Then, they carried out a perceptual experiment presenting the vowels with minimal context to listeners. When only the tokens correctly identified in the perceptual assignment were plotted in the $\left(F_{1}, F_{2}\right)$ plane, the overlap between the vowels almost disappeared. Similarly, as long as a vowel in my corpus was correctly perceived, the first two formants were sufficient to classify it.

To summarize the perceptual results so far, static information was sufficient to explain them. But we still do not know if dynamic information could be used by the listeners to identify the vowels in the perceptual task. Figure 9 shows that the parameter $a_{1}$ of the linear model (1) fitted to the second-half formant transitions could not distinguish the [a] from $[\varepsilon]$ speech signals for the subset of stimuli correctly identified in the perceptual assignment. The same results were also found for the first-half transitions and for the parameter $d$ of the nonlinear model (5) fitted to whole transitions. This suggests that the part of formant dynamics encoded in the model parameters did not play a major role in the perceptual task, i.e. the models did not isolate an important dynamic parameter for the perception.

Furthermore, the linear correlations between the perceptual scores and the medians of the dynamic parameter estimates are shown in Table VII. The correlations are lower and less consistent for the model parameters than formant measurements.

It is not perfectly clear why the dynamic parameters did not capture any feature correlated with the perceptual results. A first hypothesis is that the experiment could correspond to a situation where static information was primary used. A second hypothesis is that the dynamic models inadequately described formant dynamics or that the fitting procedures were inaccurate. Visual inspection of modeled and real formant contours suggested that dynamics was properly described by the models (see an example on Figure 4), but future work should include a study of modeling error. Because the formant values measured in the steady-state part of the vowels were sufficient to explain the perceptual results, it seems plausible that formant dynamics could have played a secondary role in the perceptual assignment. 
In summary, the first two formants measured in the steady-state parts of the vowels were highly correlated with the perceptual results and were sufficient to explain them. Conversely the dynamic parameters of the models were poorly correlated with the perceptual results and were not sufficient to explain them. This does not imply that no dynamic information was used in the perceptual task, but it does not back up dynamic specification of the vowels.

\section{CONCLUSION}

A corpus of [iai] and [i i i] was produced by two speakers in a carrier sentence at different speaking rates with two contrastive stress patterns. The corpus was perceptually analyzed, the first two formant frequencies were measured in the static parts of $[a]$ or $[\varepsilon]$, and the formant time series were dynamically and kinematically modeled. Relationships between the perceptual results, the formant measurements and the model parameters were assessed.

The analysis of the first two formant frequencies measured in the steady-state part of the vowels showed a contextual assimilation instead of a centralization effect. The same data also suggests that the speakers did not manage their contrastive stress in the same way.

The analysis of model parameters showed that dynamic or kinematic modeling led to the same results. The formant transitions were sharper for high speaking rate, but no systematic trends were found for contrastive stress.

Relationships between the perceptual scores and the formants measurements or model parameters did not support the importance of dynamic specification of vowels.

\section{ACKNOWLEDGMENTS}

This research was mainly supported by a grant from the Fondation Van Buuren and mainly carried out in the Institute of Modern Languages and Phonetics in Bruxelles. Some parts of the work were also done in the Institute of Phonetics in Aix-en-Provence with the support of a TMR grant from the European Community, and at Queen's University in 
Kingston, Canada with the support of a Natural Sciences and Engineering Research Council grant from the Government of Canada No 38842943.

I am grateful to Jean Caelen and Jean-Luc Schwartz for selecting the subject and for their valuable suggestions. I also thank Jean Schoentgen for his helpful discussions, as well as Anders Lofqvist and three anonymous referees for their important suggestions. I express my gratitude to Kevin Munhall and Jeff Jones for their pertinent and constructive comments and for translating my personal English into a real language. 


\section{REFERENCES}

Andruski, J. E. and Nearey, T. M. (1992), 'On the sufficiency of compound target specification of isolated vowels and vowels in /bVb/ syllables', The Journal of the Acoustical Society of America 91(1), 390-410.

Atal, B. S. and Hanauer, S. L. (1971), 'Speech analysis and synthesis by linear prediction of the speech wave', The Journal of the Acoustical Society of America 50(2 (Part 2)), 637-655.

Beautemps, D. (1993), Récupération des gestes de la parole à partir de trajectoires formantiques: Identification de cibles vocaliques non-atteintes et modèles pour les profils sagittaux des consonnes fricatives, PhD thesis, INPG, Grenoble.

Broad, D. J. and Clermont, F. (1987), 'A methodology for modeling vowel formant contours in CVC context', The Journal of the Acoustical Society of America 81(1), 155-165.

Carré, R., Chennoukh, S., Jospa, P. and Maeda, S. (1996), The ears are not sensitive to certain coarticulatory variations: Results from VCV synthesis/perceptual experiments, in '1st ESCA Tutorial and Research Workshop on Speech Production Modeling: From Control Strategies to Acoustics', European Speech Communication Association, Autrans, France, pp. $13-16$.

Carré, R., Chennoukh, S., Lindblom, B. and Divenyi, P. (1994), 'On the perceptual caracteristics of "speech gestures"', The Journal of the Acoustical Society of America 96, 3326-3326. ASA meeting held in Austin.

Carré, R. and Mrayati, M. (1992), 'Distinctive regions in acoustic tubes. speech production modelling', Journal of Acoustique 5, 141-159.

Cassidy, S. and Harrington, J. (1992), Investigating the dynamic structure of vowels using neural networks, in 'Proceedings of the Fourth Australian International Conference on Speech Science and Technology', Australian Speech Science and Technology Association, University of Queensland, Brisbane, Australia, pp. 495-500. 
Delattre, P., Liberman, A. M., Cooper, F. S. and Gerstman, L. J. (1952), 'An experimental study of the acoustic determinants of vowel color; observations on one- and two-formant vowels synthesized from spectrographic patterns', Word 8, 195-210.

Divenyi, P., Lindblom, B. and Carré, R. (1995), The role of transition velocity in the perception of $\mathrm{V}_{1} \mathrm{~V}_{2}$ complexes, in Elenius and Branderud (1995), pp. 258-261.

Elenius, K. and Branderud, P., eds (1995), Proceedings of the XIIIth International Congress of Phonetic Sciences, Department of Speech Communication and Music Acoustics, KTH (Royal Institute of Technology), and the Department of Linguistics, Stockholm University, Stockholm, Sweden.

Gay, T., Ushijima, T., Hirose, H. and Cooper, F. S. (1974), 'Effect of speaking rate on labial consonant-vowel articulation', Journal of Phonetics 2, 47-63.

Guenther, F. H. (1995), 'Speech sound acquisition, coarticulation, and rate effects in a neural network model of speech production', Psychological Review 102(3), 594-621.

Helmholtz, H. F. (1885 - 1954), On the Sensations of Tone, (Translated by A. J. Ellis. Reprinted in 1954). New York: Dover.

Imaizumi, S. and Kiritani, S. (1989), 'Effect of speaking rate on formant trajectories and inter-speaker variations', Ann. Bull. RILP 23, 27-37.

Jenkins, J. J., Strange, W. and Trent, S. A. (1999), 'Context-independent dynamic information for the perception of coarticulated vowels', The Journal of the Acoustical Society of America 106(1), 438-448.

Joos, M. (1948), Acoustic phonetics, in L. S. of America, ed., 'Language Monograph No 23', Baltimore, MD: Waverly.

Koopmans-van Beinum, F. J. (1980), Vowel contrast reduction, an acoustic and perceptual study of Dutch vowels in various speech conditions, PhD thesis, University of Amsterdam. 
Kuehn, D. P. and Moll, K. L. (1976), 'A cineradiographic study of VC and CV articulatory velocities', Journal of Phonetics 4, 303-320.

Kuwabara, H. (1985), 'An approach to normalization of coarticulation effects for vowels in connected speech', The Journal of the Acoustical Society of America 77(2), 686-694.

Laboissière, R., Ostry, D. and Perrier, P. (1995), A model of human jaw and hyoid motion and its implications for speech production, in Elenius and Branderud (1995), pp. 60-67.

Lindblom, B. E. F. (1963), 'Spectrographic study of vowel reduction', The Journal of the Acoustical Society of America 35(11), 1773-1781.

Lindblom, B. E. F. and Studdert-Kennedy, M. (1967), 'On the role of formant transitions on vowel recognition', The Journal of the Acoustical Society of America 42(4), 830-843.

Loevenbruck, H. and Perrier, P. (1993), Vocalic reduction : Prediction of acoustic and articulatory variabilities with invariant motor commands, in 'Proceedings of Eurospeech'93', Vol. 1, catalyst consult, ESCA, Berlin, Germany, pp. 85-88.

Majid, R., Abry, C., Boe, L.-J. and Perrier, P. (1987), 'Fonctions de macro-sensibilité articulatori-acoustique, pour le système vocalique du français, à partir d'un modèle articulatoire du conduit vocal', Bulletin du Laboratoire de la Communication Parlée de Grenoble 1A, 133-157.

Makhoul, J. (1975), 'Linear prediction: A tutoral review', Proceedings of the IEEE $63(4), 561-580$.

Marchal, A. and Meynadier, Y. (1996), Coarticulation in /kl/ sequences in French: a multisensor investigation of the timing of lingual gestures, in 'Proceedings of the 6th Australian International Conference on Speech Science and Technology', Australian Speech Science and Technology Association inc, Adelaide, Australia, pp. 434-438.

Moon, S.-J. and Lindblom, B. (1994), 'Interaction between duration, context, and speaking 
style in English stressed vowels', The Journal of the Acoustical Society of America 96(1), 4055.

Nábĕlek, A. K. and Ovchinnikov, A. (1997), 'Perception of nonlinear and linear formant trajectories', The Journal of the Acoustical Society of America 101(1), 488-497.

Nearey, T. M. (1989), 'Static, dynamic, and relational properties in vowel perception', The Journal of the Acoustical Society of America 85(5), 2088-2113.

Nearey, T. M. and Assmann, P. F. (1986), 'Modeling the role of inherent spectral change in vowel identification', The Journal of the Acoustical Society of America 80(5), 1297-1308.

Peterson, G. and Barney, H. (1952), 'Control methods used in a study of vowels', The Journal of the Acoustical Society of America 24, 175-184.

Pitermann, M. (1996), Évaluation expérimentale de la théorie des cibles formantiques dans le cadre de la production des voyelles, PhD thesis, Université Libre de Bruxelles.

Polka, L. and Bohn, O.-S. (1996), 'A cross-language comparison of vowel perception in English-learning and German-learning infants', The Journal of the Acoustical Society of America 100(1), 577-592.

Pols, L. C. W. and van Son, R. J. J. H. (1993), 'Acoustics and perception of dynamic vowel segments', Speech Communication 13, 135-147.

Press, W. H., Teukolsky, S. A., Vetterling, W. T. and Flannery, B. P. (1992), Numerical Recipes in $C$, second edn, Cambridge University Press, chapter 15: Modeling of Data, pp. $681-688$.

Sonoda, Y. (1987), 'Effect of speaking rate on articulatory dynamics and motor event', Journal of Phonetics 15, 145-156.

Soquet, A. (1995a), A cooperative approach to formant extraction, in Elenius and Branderud (1995), pp. 448-451. 
Soquet, A. (1995b), Étude comparée de représentations acoustiques et articulatoires du signal de parole pour le décodage acoustico-phonétique. Application à la classification de voyelles et à la détermination du lieu d'articulation des occlusives, $\mathrm{PhD}$ thesis, Université Libre de Bruxelles.

Spiegel, M. R. (1988), Statistics, 2/ed, Schaum's Outline Series, McGraw-Hill, Inc.

Stevens, K. N. and House, A. S. (1963), 'Perturbation of vowel articulations by consonantal context: an acoustical study', Journal of Speech and Hearing Research 6(2), 111-128.

Strange, W. (1989a), 'Dynamic specification of coarticulated vowels spoken in sentence context', The Journal of the Acoustical Society of America 85(5), 2135-2153.

Strange, W. (1989b), 'Evolving theories of vowel perception', The Journal of the Acoustical Society of America 85(5), 2081-2087.

Strange, W. and Bohn, O.-S. (1998), 'Dynamic specification of coarticulated German vowels: Perceptual and acoustical studies', The Journal of the Acoustical Society of America 104(1), 488-504.

van Son, R. J. J. H. (1993), Vowel perception: a closer look at the literature, in 'Proceedings of the Institute of Phonetic Sciences, University of Amsterdam', Vol. 17, pp. 33-64.

van Son, R. J. J. H. and Pols, L. C. W. (1992), 'Formant movements of Dutch vowels in a text, read at normal and fast rate', The Journal of the Acoustical Society of America 92(1), 121-127.

Viterbi, A. (1967), 'Error bounds for convolutional codes and an asymptotically optimum decoding algorithm', IEEE Trans. formation Theory IT-13, 260-269.

Watson, C. I. and Harrington, J. (1999), 'Acoustic evidence for dynamic formant trajectories in Australian English vowels', The Journal of the Acoustical Society of America 106(1), 458468. 


\section{TABLES}

TABLE I. Equations of the dynamic and kinematic models. Symbol $x_{n}$ represents the formant frequency at time coordinate $n \Delta t ; a, a_{i}, b, c, c_{1}, c_{2}, d, p, q, t_{0}$, and $x^{*}$ are the model parameters; $k$ is the order of linear model (1); $\Delta t$ is the sampling step (i.e. $9 \mathrm{~ms}$ ).

\begin{tabular}{|c|c|c|c|c|}
\hline & dynamic models & & kinematic models & \\
\hline linear \& exp. & $x_{n}=a_{0}+\sum_{i=1}^{k} a_{i} x_{n-i}$ & $(1)$ & $x_{n}=x^{*}+p e^{-q n \Delta t}$ & $(2)$ \\
\hline critical damping & $x_{n}=a_{0}+a_{1} x_{n-1}-\frac{a_{1}^{2}}{4} x_{n-2}$ & $(3)$ & $x_{n}=x^{*}+\left(c_{1}+c_{2} n \Delta t\right) e^{-q n \Delta t}$ & $(4)$ \\
\hline logistic & $x_{n}=c+\frac{a\left(x_{n-1}-c\right)}{a d+(1-d)\left(x_{n-1}-c\right)}$ & $(5)$ & $x_{n}=c+\frac{a}{1+e^{-b\left(n \Delta t-t_{0}\right)}}$ & (6) \\
\hline
\end{tabular}


TABLE II. Medians and semi-68-\% ranges of the first two formant frequencies (Hz) measured in the steady-state part of speakers' $[\mathrm{i}],[\varepsilon]$ or $[\mathrm{a}]$. The medians were computed across all speaking $\underline{\text { rate and stress conditions. }}$

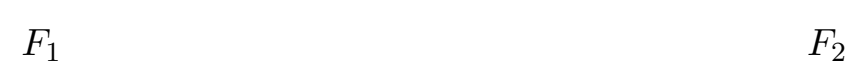

\begin{tabular}{lrrrrrrrr} 
& \multicolumn{2}{c}{ Speaker A } & \multicolumn{2}{c}{ Speaker B } & \multicolumn{2}{c}{ Speaker A } & \multicolumn{2}{c}{ Speaker B } \\
& median & range & median & range & median & range & median & range \\
\hline$[\mathrm{i}]$ & 282 & 28 & 225 & 14 & 1965 & 36 & 1954 & 43 \\
{$[\varepsilon]$} & 373 & 20 & 353 & 32 & 1848 & 37 & 1890 & 52 \\
{$[\mathrm{a}]$} & 530 & 73 & 513 & 144 & 1661 & 102 & 1732 & 168 \\
\hline \hline
\end{tabular}


TABLE III. Results of analyses of variance of formant measurements for speaking rate and contrastive stress factors. One analysis was carried out for each combination of formant, speaker and vowel. Labels "DF", "s:r" and "resid" stand for degree of freedom, interaction between stress and rate, and residuals respectively. When $p<1 \mathrm{e}-10,0$ was reported in the table.

\section{$F_{1}$}

Speaker A

[E]

Speaker B

\begin{tabular}{|c|c|c|c|c|c|c|c|c|c|c|c|c|}
\hline & & {$[\varepsilon]$} & & & [a] & & & {$[\varepsilon]$} & & & [a] & \\
\hline & $\mathrm{DF}$ & $F$ & $p$ & $\mathrm{DF}$ & $F$ & $p$ & $\mathrm{DF}$ & $F$ & $p$ & $\mathrm{DF}$ & $F$ & $p$ \\
\hline stress & 1 & 17.3 & $3.7 \mathrm{e}-5$ & 1 & 79.9 & 0 & 1 & 296.3 & 0 & 1 & 4261.3 & 0 \\
\hline rate & 9 & 11.4 & 0 & 9 & 158.4 & 0 & 8 & 47.1 & 0 & 8 & 637.1 & 0 \\
\hline $\mathrm{s}: \mathrm{r}$ & 9 & 24.6 & 0 & 9 & 3.1 & 0.0011 & 8 & 11.8 & 0 & 8 & 83.7 & 0 \\
\hline resid & 604 & & & 620 & & & 569 & & & 589 & & \\
\hline
\end{tabular}

$F_{2}$

Speaker A

[E]

DF $\quad F$ [a]

Speaker B

\begin{tabular}{|c|c|c|c|c|c|c|c|c|c|c|c|c|}
\hline & $\mathrm{DF}$ & $F$ & $p$ & $\mathrm{DF}$ & $F$ & $p$ & $\mathrm{DF}$ & $F$ & $p$ & $\mathrm{DF}$ & $F$ & $p$ \\
\hline stress & 1 & 37.5 & $1.7 \mathrm{e}-9$ & 1 & 34.4 & $7.4 \mathrm{e}-9$ & 1 & 549.4 & 0 & 1 & 558.3 & 0 \\
\hline rate & 9 & 52.6 & 0 & 9 & 489.4 & 0 & 8 & 81.6 & 0 & 8 & 972.5 & 0 \\
\hline s:r & 9 & 10.0 & 0 & 9 & 8.3 & 0 & 8 & 10.7 & 0 & 8 & 44.2 & 0 \\
\hline resid & 599 & & & 620 & & & 553 & & & 584 & & \\
\hline
\end{tabular}


TABLE IV. Results of analyses of variance of $a_{1}$ (linear model (1)) estimates fitted to the first-half formant transitions for speaking rate and contrastive stress factors. One analysis was carried out for each combination of formant, speaker and vowel. Labels "DF", "s:r" and "resid" stand for degree of freedom, interaction between stress and rate, and residuals respectively. When $p<1 \mathrm{e}-10,0$ was reported in the table.

\section{$F_{1}$}

Speaker A Speaker B

\begin{tabular}{|c|c|c|c|c|c|c|c|c|c|c|c|c|}
\hline & & {$[\varepsilon]$} & & & {$[\mathrm{a}]$} & & & {$[\varepsilon]$} & & & [a] & \\
\hline & $\mathrm{DF}$ & $F$ & $p$ & $\mathrm{DF}$ & $F$ & $p$ & $\mathrm{DF}$ & $F$ & $p$ & $\mathrm{DF}$ & $F$ & $p$ \\
\hline stress & 1 & 0.55 & 0.46 & 1 & 20.83 & $6.3 \mathrm{e}-6$ & 1 & 0.67 & 0.41 & 1 & 68.86 & 0 \\
\hline rate & 9 & 0.76 & 0.65 & 9 & 1.98 & 0.040 & 8 & 5.51 & $1.0 \mathrm{e}-6$ & 8 & 1.77 & 0.081 \\
\hline s:r & 9 & 0.68 & 0.73 & 9 & 8.03 & 0 & 8 & 3.60 & $4.4 \mathrm{e}-4$ & 8 & 3.41 & $7.9 \mathrm{e}-4$ \\
\hline resid & 444 & & & 522 & & & 539 & & & 545 & & \\
\hline
\end{tabular}

\section{$F_{2}$}

Speaker A Speaker B

\begin{tabular}{|c|c|c|c|c|c|c|c|c|c|c|c|c|}
\hline & & {$[\varepsilon]$} & & & [a] & & & {$[\varepsilon]$} & & & [a] & \\
\hline & $\mathrm{DF}$ & $F$ & $p$ & $\mathrm{DF}$ & $F$ & $p$ & $\mathrm{DF}$ & $F$ & $p$ & $\mathrm{DF}$ & $F$ & $p$ \\
\hline stress & 1 & 0.51 & 0.47 & 1 & 0.57 & 0.45 & 1 & 9.23 & 0.0025 & 1 & 0.87 & 0.35 \\
\hline rate & 9 & 1.26 & 0.26 & 9 & 0.97 & 0.46 & 8 & 1.23 & 0.28 & 8 & 0.90 & 0.52 \\
\hline s:r & 9 & 0.76 & 0.65 & 9 & 0.93 & 0.50 & 8 & 1.30 & 0.24 & 8 & 0.82 & 0.59 \\
\hline resid & 514 & & & 502 & & & 514 & & & 561 & & \\
\hline
\end{tabular}


TABLE V. Results of analyses of variance of $a_{1}$ (linear model (1)) estimates fitted to the second-half formant transitions for speaking rate and contrastive stress factors. One analysis was carried out for each combination of formant, speaker and vowel. Labels "DF", "s:r" and "resid" stand for degree of freedom, interaction between stress and rate, and residuals respectively. When $p<1 \mathrm{e}-10,0$ was reported in the table.

\section{$F_{1}$}

Speaker A Speaker B

\begin{tabular}{|c|c|c|c|c|c|c|c|c|c|c|c|c|}
\hline & & {$[\varepsilon]$} & & & {$[\mathrm{a}]$} & & & {$[\varepsilon]$} & & & {$[\mathrm{a}]$} & \\
\hline & $\mathrm{DF}$ & $F$ & $p$ & $\mathrm{DF}$ & $F$ & $p$ & $\mathrm{DF}$ & $F$ & $p$ & $\mathrm{DF}$ & $F$ & $p$ \\
\hline stress & 1 & 3.42 & 0.065 & 1 & 0.75 & 0.39 & 1 & 2.48 & 0.12 & 1 & 51.80 & 0 \\
\hline rate & 9 & 2.35 & 0.013 & 9 & 1.20 & 0.29 & 8 & 1.29 & 0.24 & 8 & 18.12 & 0 \\
\hline s:r & 9 & 1.99 & 0.038 & 9 & 1.06 & 0.39 & 8 & 2.67 & 0.0069 & 8 & 8.88 & 0 \\
\hline resid & 570 & & & 569 & & & 548 & & & 538 & & \\
\hline
\end{tabular}

$F_{2}$

Speaker A Speaker B

\begin{tabular}{|c|c|c|c|c|c|c|c|c|c|c|c|c|}
\hline & & {$[\varepsilon]$} & & & [a] & & & {$[\varepsilon]$} & & & [a] & \\
\hline & $\mathrm{DF}$ & $F$ & $p$ & $\mathrm{DF}$ & $F$ & $p$ & $\mathrm{DF}$ & $F$ & $p$ & $\mathrm{DF}$ & $F$ & $p$ \\
\hline stress & 1 & 2.89 & 0.089 & 1 & 0.88 & 0.35 & 1 & 0.46 & 0.50 & 1 & 3.11 & 0.079 \\
\hline rate & 9 & 1.35 & 0.21 & 9 & 1.80 & 0.066 & 8 & 1.62 & 0.12 & 8 & 3.90 & $1.8 \mathrm{e}-4$ \\
\hline s:r & 9 & 0.99 & 0.45 & 9 & 1.57 & 0.12 & 8 & 0.85 & 0.56 & 8 & 1.72 & 0.091 \\
\hline resid & 554 & & & 596 & & & 385 & & & 448 & & \\
\hline
\end{tabular}


TABLE VI. Results of analyses of variance of $d$ (nonlinear model (5)) estimates fitted to complete formant transitions for speaking rate and contrastive stress factors. One analysis was carried out for each combination of formant, speaker and vowel. Labels "DF", "s:r" and "resid" stand for degree of freedom, interaction between stress and rate, and residuals respectively. When $p<1 \mathrm{e}-10,0$ was reported in the table.

\section{$F_{1}$}

Speaker A Speaker B

\begin{tabular}{|c|c|c|c|c|c|c|c|c|c|c|c|c|}
\hline & & {$[\varepsilon]$} & & & {$[\mathrm{a}]$} & & & {$[\varepsilon]$} & & & {$[\mathrm{a}]$} & \\
\hline & $\mathrm{DF}$ & $F$ & $p$ & $\mathrm{DF}$ & $F$ & $p$ & $\mathrm{DF}$ & $F$ & $p$ & $\mathrm{DF}$ & $F$ & $p$ \\
\hline stress & 1 & 1.37 & 0.24 & 1 & 1.23 & 0.27 & 1 & 2.26 & 0.13 & 1 & 237.16 & 0 \\
\hline rate & 9 & 12.70 & 0 & 9 & 20.63 & 0 & 8 & 8.02 & 0 & 8 & 2.68 & 0.0067 \\
\hline s:r & 9 & 3.77 & $1.2 \mathrm{e}-4$ & 9 & 10.89 & 0 & 8 & 7.57 & 0 & 8 & 6.51 & $3.9 \mathrm{e}-8$ \\
\hline resid & 604 & & & 615 & & & 567 & & & 586 & & \\
\hline
\end{tabular}

$F_{2}$

Speaker A Speaker B

\begin{tabular}{|c|c|c|c|c|c|c|c|c|c|c|c|c|}
\hline & & {$[\varepsilon]$} & & & [a] & & & {$[\varepsilon]$} & & & {$[\mathrm{a}]$} & \\
\hline & $\mathrm{DF}$ & $F$ & $p$ & $\mathrm{DF}$ & $F$ & $p$ & $\mathrm{DF}$ & $F$ & $p$ & $\mathrm{DF}$ & $F$ & $p$ \\
\hline stress & 1 & 14.84 & $1.3 \mathrm{e}-4$ & 1 & 0.037 & 0.85 & 1 & 2.09 & 0.15 & 1 & 0.99 & 0.32 \\
\hline rate & 9 & 5.05 & $1.4 \mathrm{e}-6$ & 9 & 18.07 & 0 & 8 & 1.46 & 0.17 & 8 & 1.17 & 0.32 \\
\hline s:r & 9 & 2.31 & 0.015 & 9 & 1.68 & 0.091 & 8 & 0.56 & 0.81 & 8 & 1.05 & 0.40 \\
\hline resid & 602 & & & 616 & & & 545 & & & 568 & & \\
\hline
\end{tabular}


TABLE VII. Standard linear Pearson correlations between the medians of the formant measurements or the medians of the model parameters and the perceptual results for [a]. The medians are shown in Figures 4, 5, 6 and 7, the perceptual scores are shown in Figure 3. Labels "u" or "s" stands for unstressed or stressed [a], " $F_{i}$ " for the $i$ th formant, " $a_{1}(1)$ " or " $a_{1}(2)$ " for the parameter $a_{1}$ of the linear model (1) estimated on the first- or second-half transitions, and $d$ for the parameter $d$ of the logistic model (5). Correlations printed in bold were significantly different from 0 at 0.05 level according to a two-tail standard Pearson sample correlation test. The number of degrees of freedom were 8 and 7 respectively for speaker $\mathrm{A}$ and $\mathrm{B}$.

\begin{tabular}{|c|c|c|c|c|c|c|c|c|}
\hline & \multicolumn{4}{|c|}{$F_{1}$} & \multicolumn{4}{|c|}{$F_{2}$} \\
\hline & \multicolumn{2}{|c|}{ Speaker A } & \multicolumn{2}{|c|}{ Speaker B } & \multicolumn{2}{|c|}{ Speaker A } & \multicolumn{2}{|c|}{ Speaker B } \\
\hline & $\mathrm{u}$ & $\mathrm{s}$ & $\mathrm{u}$ & $\mathrm{s}$ & $\mathrm{u}$ & $\mathrm{s}$ & $\mathrm{u}$ & $\mathrm{s}$ \\
\hline$F_{i}$ & 0.82 & 0.87 & 0.90 & 0.89 & -0.85 & -0.87 & -0.94 & -0.81 \\
\hline$a_{1}(1)$ & -0.52 & 0.84 & 0.35 & 0.74 & -0.73 & -0.21 & 0.75 & 0.86 \\
\hline$a_{1}(2)$ & 0.79 & 0.46 & 0.02 & 0.49 & 0.89 & 0.96 & 0.68 & 0.53 \\
\hline$d$ & 0.79 & 0.51 & -0.22 & -0.78 & 0.83 & 0.82 & 0.68 & -0.56 \\
\hline
\end{tabular}




\section{FIGURES}

FIG. 1. Plots of each kinematic model (solid lines) fitted to a second-half or full transition of the first formant (dotted line) of the first part of a [iai] stimulus.

FIG. 2. Length medians of the [ia] or [i $\varepsilon] F_{1}$ transitions plotted as functions of speaking rate measured in metronome rhythm: 'O' stressed vowels, ' $\times$ ' unstressed vowels, solid lines for [ia], and dotted lines for $[i \varepsilon]$. Low speaking rates are on the left hand side of each graph.

FIG. 3. Normalized identification scores averaged over the seven listeners for speakers A and B's vowel [a] as functions of speaking rate: 'O' stressed [a], ' $x$ ' unstressed [a]. Low speaking rates are on the left hand side of each graph.

FIG. 4. Medians of the first two formant frequencies $(\mathrm{Hz})$ measured in the steady-state part of speakers' $[\varepsilon]$ and $[\mathrm{a}]$ for all combinations of speaking rate and contrastive stress. The data is plotted as functions of speaking rate measured in metronome rhythm: 'O' stressed vowels, ' $X$ ' unstressed vowels, solid lines for $[\mathrm{a}]$, and dotted lines for $[\varepsilon]$. Low speaking rates are on the left hand side of each graph.

FIG. 5. Medians of parameter $a_{1}$ of the linear model (1) fitted to the first-half formant transitions of speakers' [i $\varepsilon]$ or [ia] for each combination of speaking rate and contrastive stress. The data is plotted as functions of speaking rate measured in metronome rhythm: 'O' stressed vowels, ' $X$ '

unstressed vowels, solid lines for $[\mathrm{a}]$, and dotted lines for $[\varepsilon]$. Low speaking rates are on the left hand side of each graph.

FIG. 6. Medians of parameter $a_{1}$ of the linear model (1) fitted to the second-half formant transitions of speakers' [i $\varepsilon$ ] or [ia] for each combination of speaking rate and contrastive stress. The data is plotted as functions of speaking rate measured in metronome rhythm: 'O' stressed vowels, ' $\times$ ' unstressed vowels, solid lines for $[\mathrm{a}]$, and dotted lines for $[\varepsilon]$. Low speaking rates are on the left hand side of each graph. 
FIG. 7. Medians of parameter $d$ of the logistic model (5) fitted to the whole formant transitions of speakers' [i $\varepsilon]$ or [ia] for each combination of speaking rate and contrastive stress. The data is plotted as functions of speaking rate measured in metronome rhythm: 'O' stressed vowels, ' $X$ ' unstressed vowels, solid lines for $[a]$, and dotted lines for $[\varepsilon]$. Low speaking rates are on the left hand side of each graph.

FIG. 8. Formant measurements for the $[\mathrm{a}]$ and $[\varepsilon]$ correctly identified in the perceptual experiment. The 'l' signs correspond to the $[\varepsilon]$, the '-' to the [a]. The axes are linearly graduated in Hz.

FIG. 9. Parameter $a_{1}$ of the linear model (1) fitted to the second-half $F_{1}$ or $F_{2}$ transitions of the [ia] or [i $\varepsilon$ ] stimuli correctly identified in the perceptual experiment. The $x$ axis corresponds to $F_{1}$, the $y$ axis to $F_{2}$, the '|' signs to $[\varepsilon]$ and '-' to [a] stimuli. 
FIG. 1. Michel Pitermann, The Journal of the Acoustical Society of America.
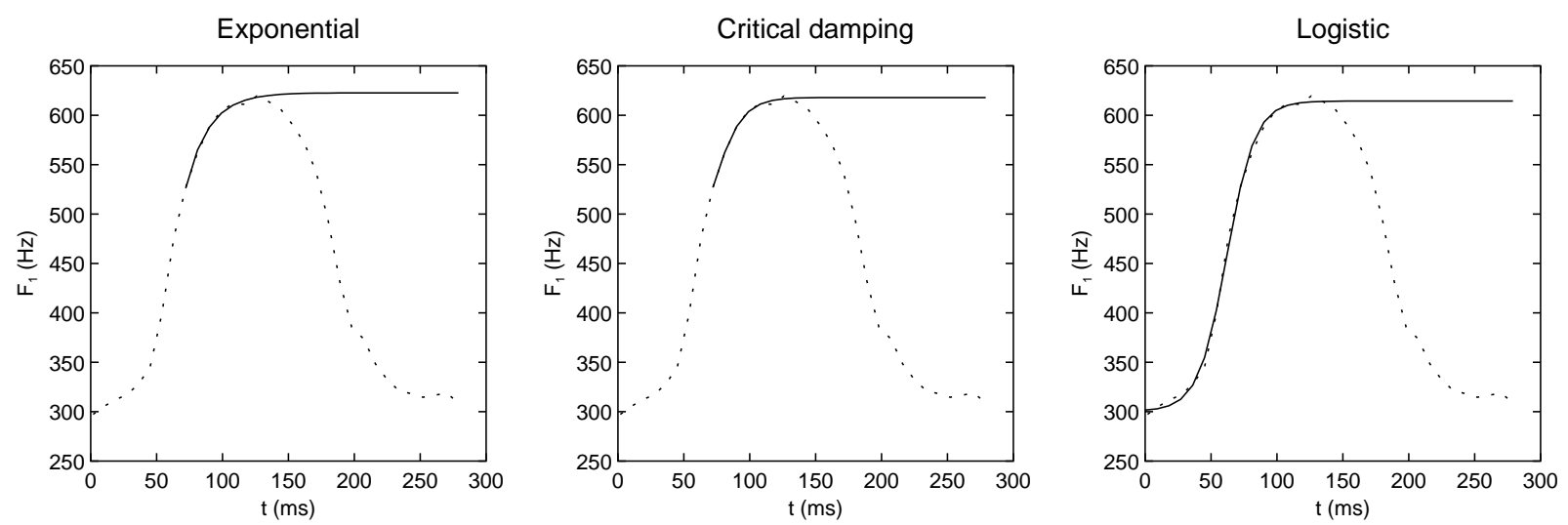
FIG. 2. Michel Pitermann, The Journal of the Acoustical Society of America.
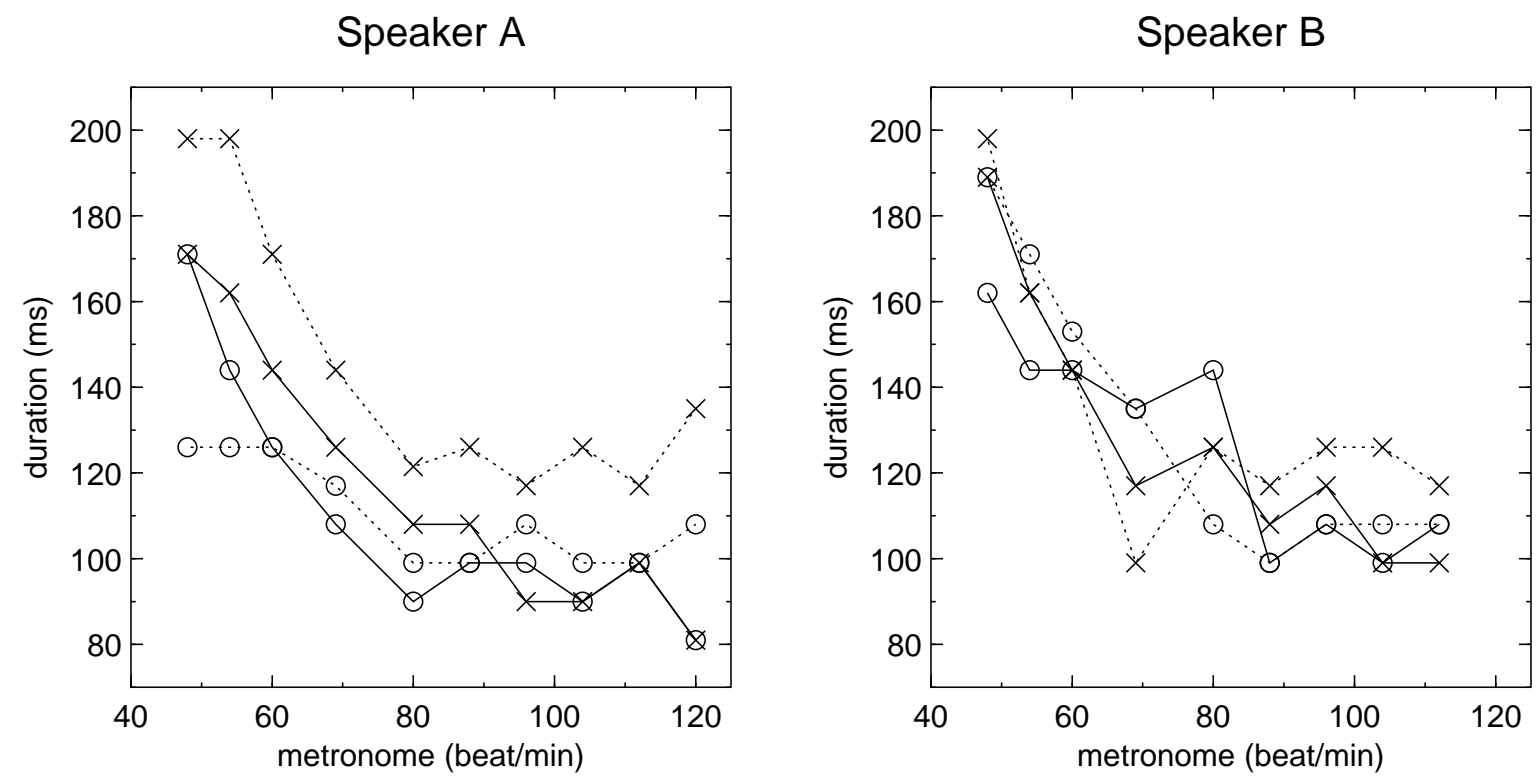
FIG. 3. Michel Pitermann, The Journal of the Acoustical Society of America.
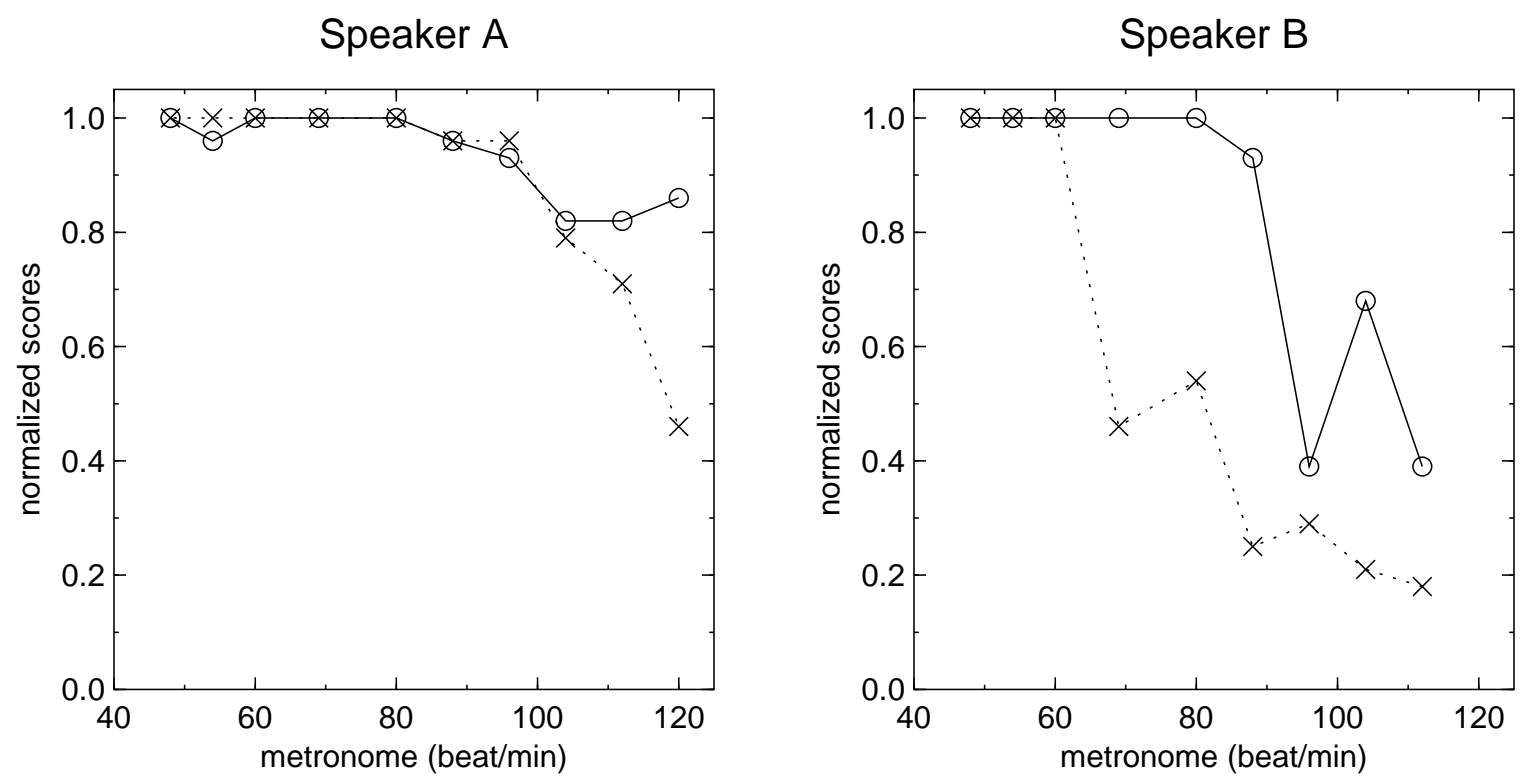
FIG. 4. Michel Pitermann, The Journal of the Acoustical Society of America.
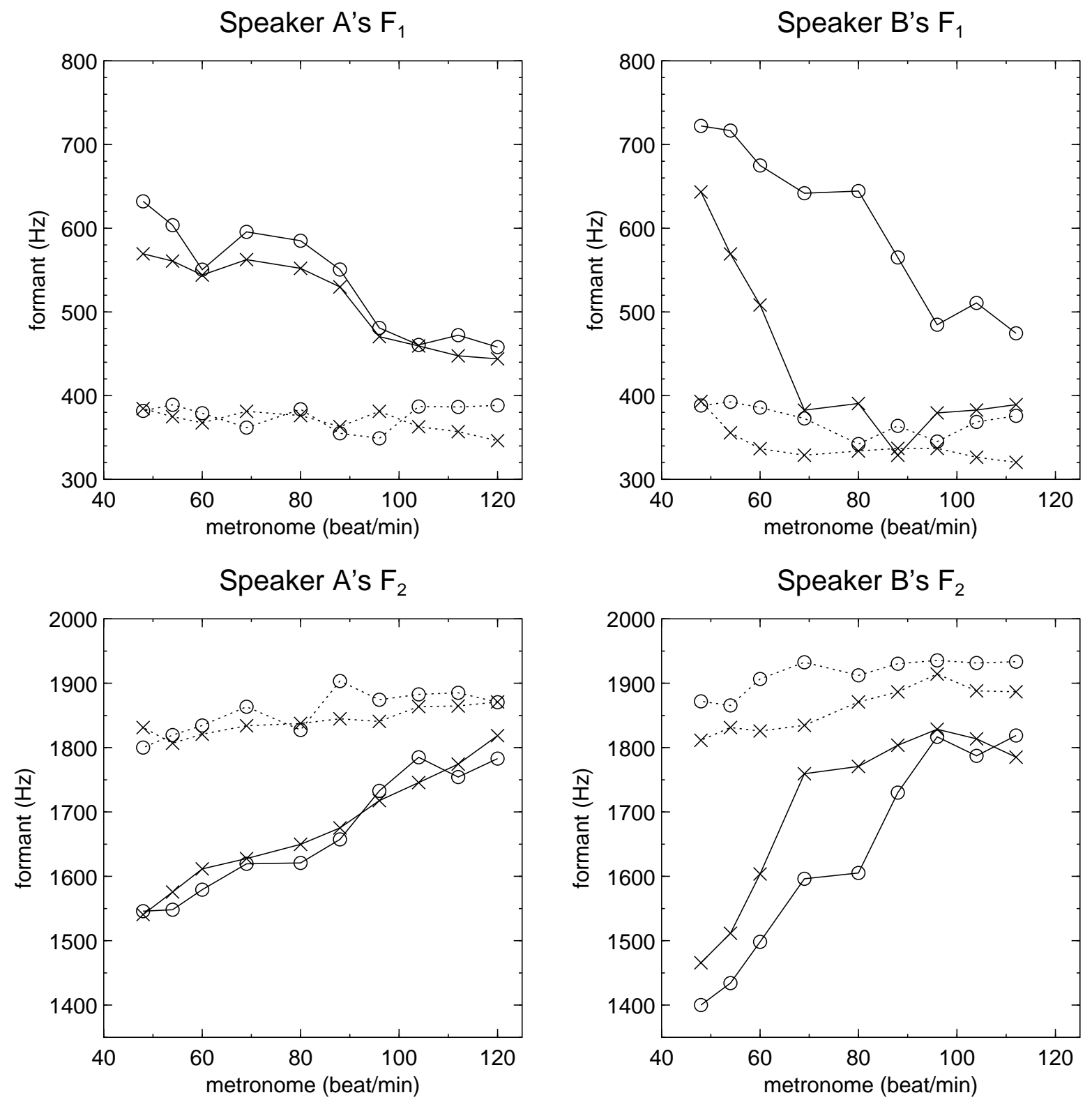
FIG. 5. Michel Pitermann, The Journal of the Acoustical Society of America.
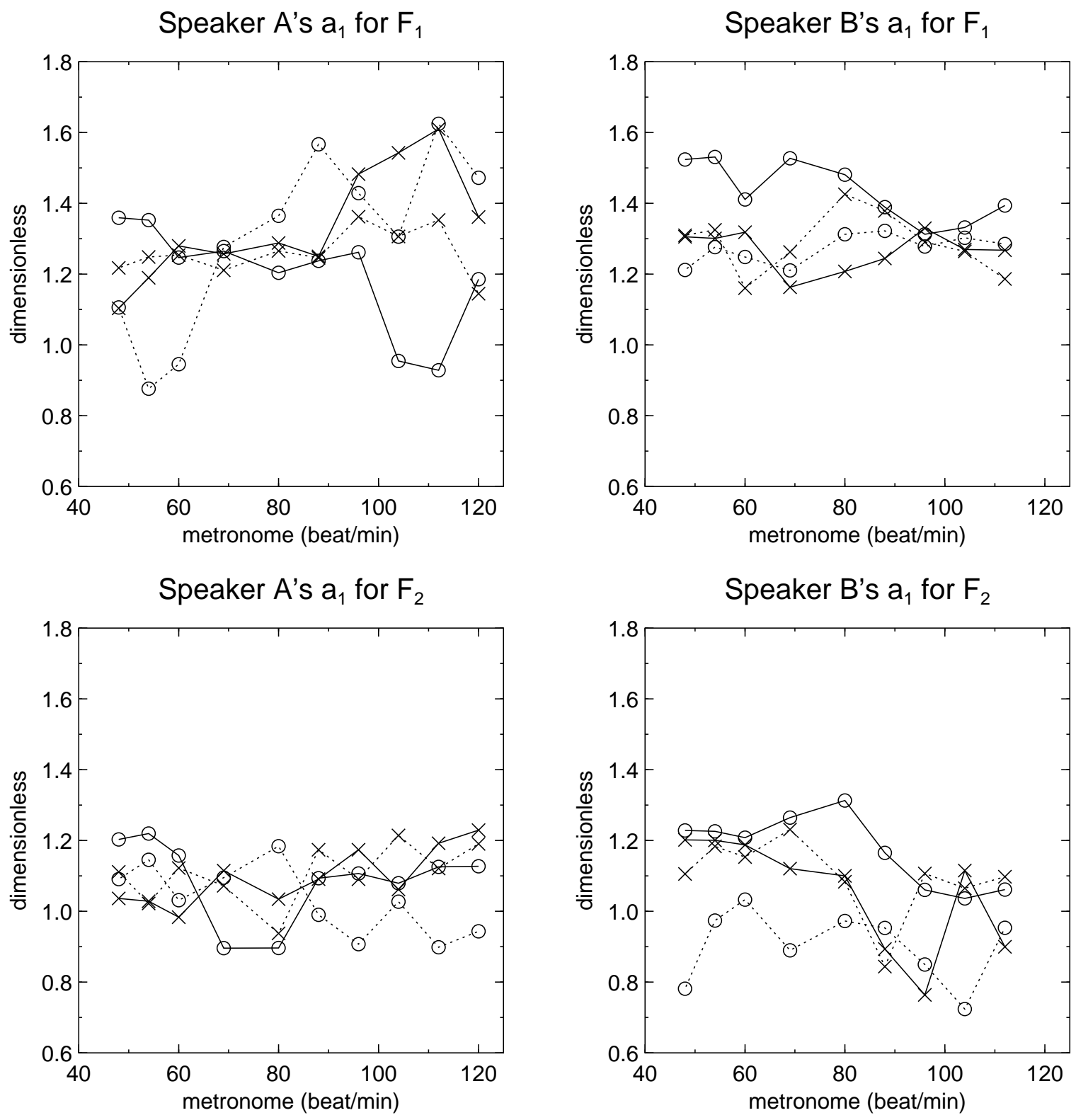
FIG. 6. Michel Pitermann, The Journal of the Acoustical Society of America.
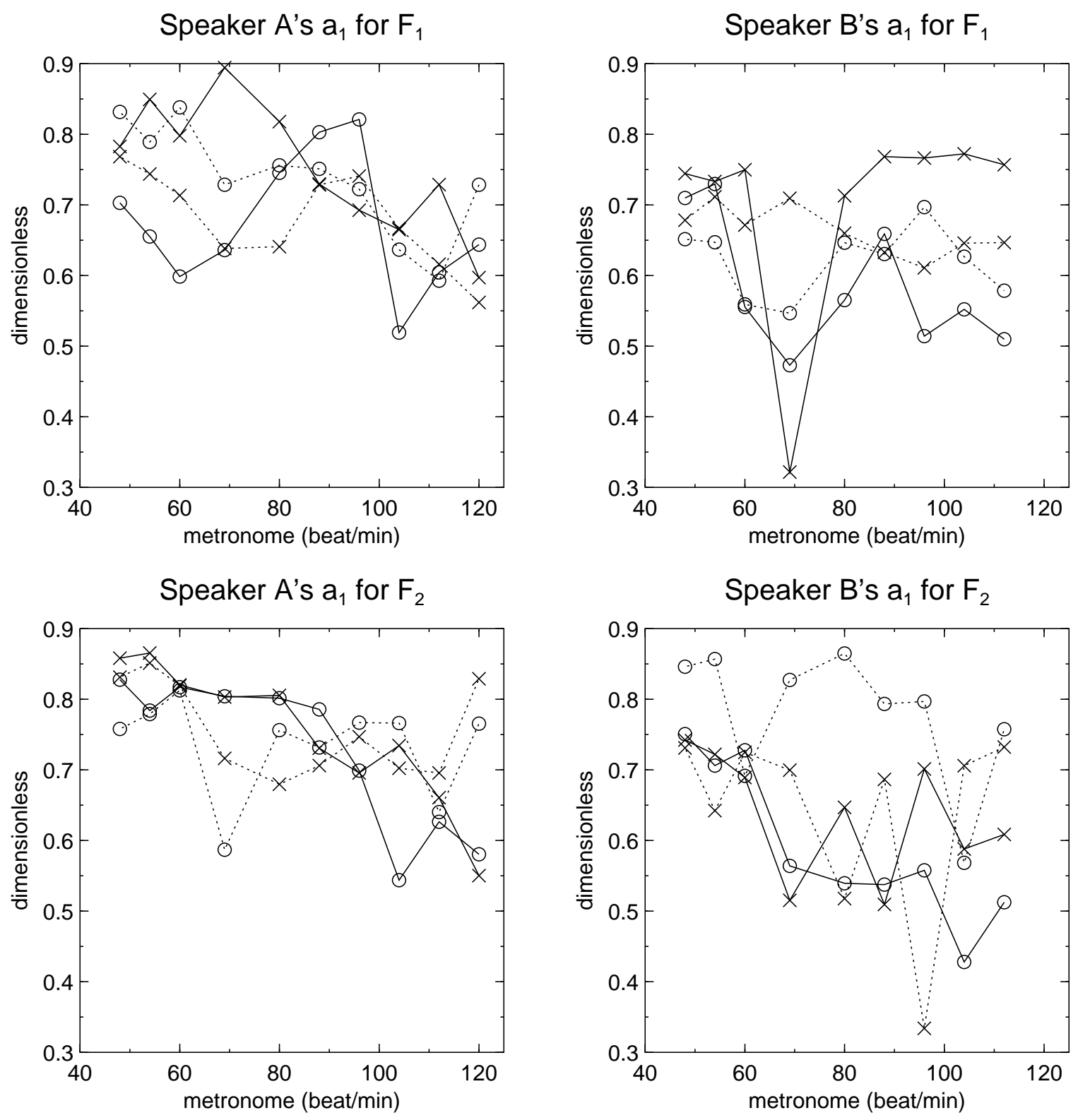
FIG. 7. Michel Pitermann, The Journal of the Acoustical Society of America.
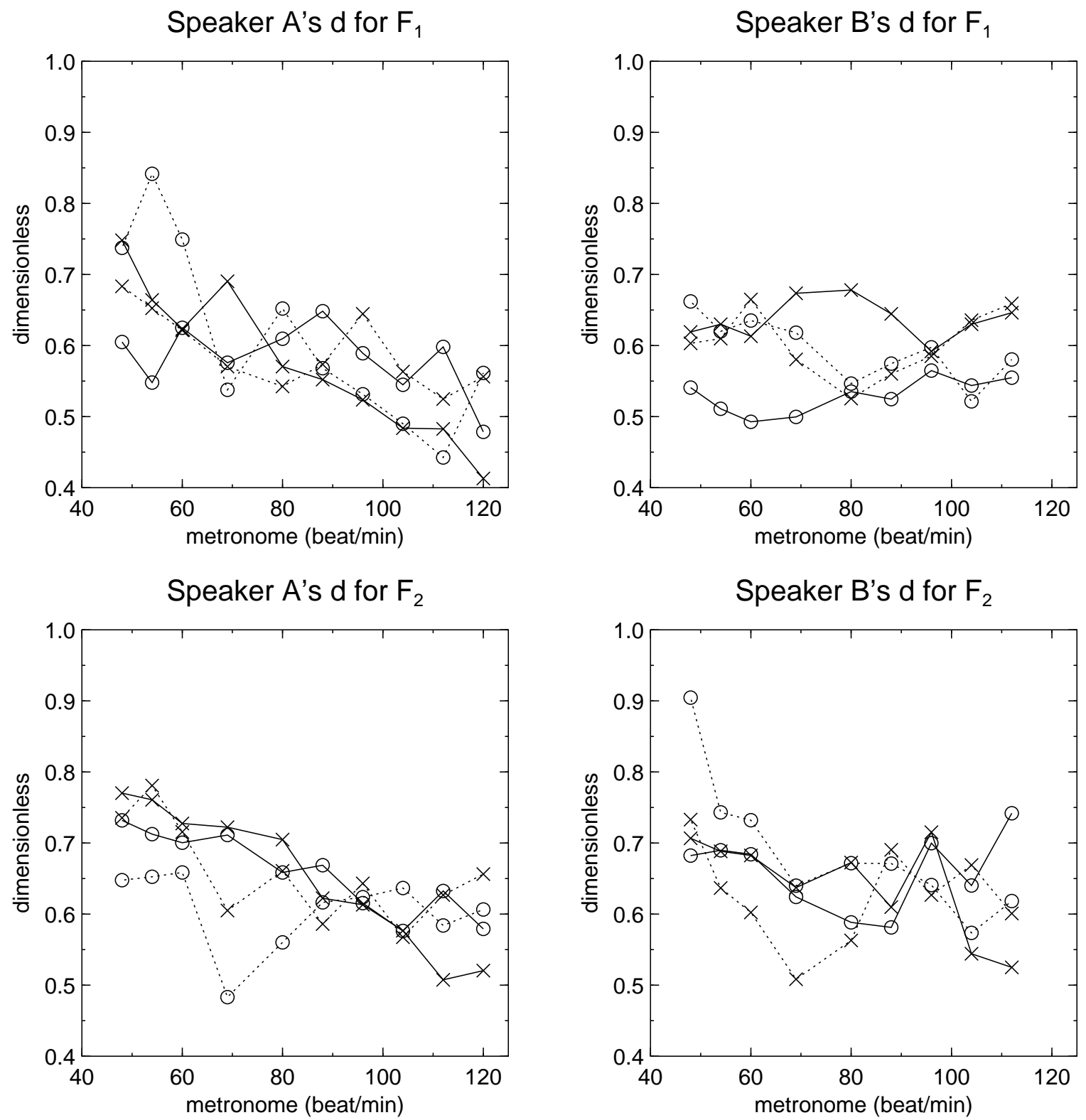
FIG. 8. Michel Pitermann, The Journal of the Acoustical Society of America.

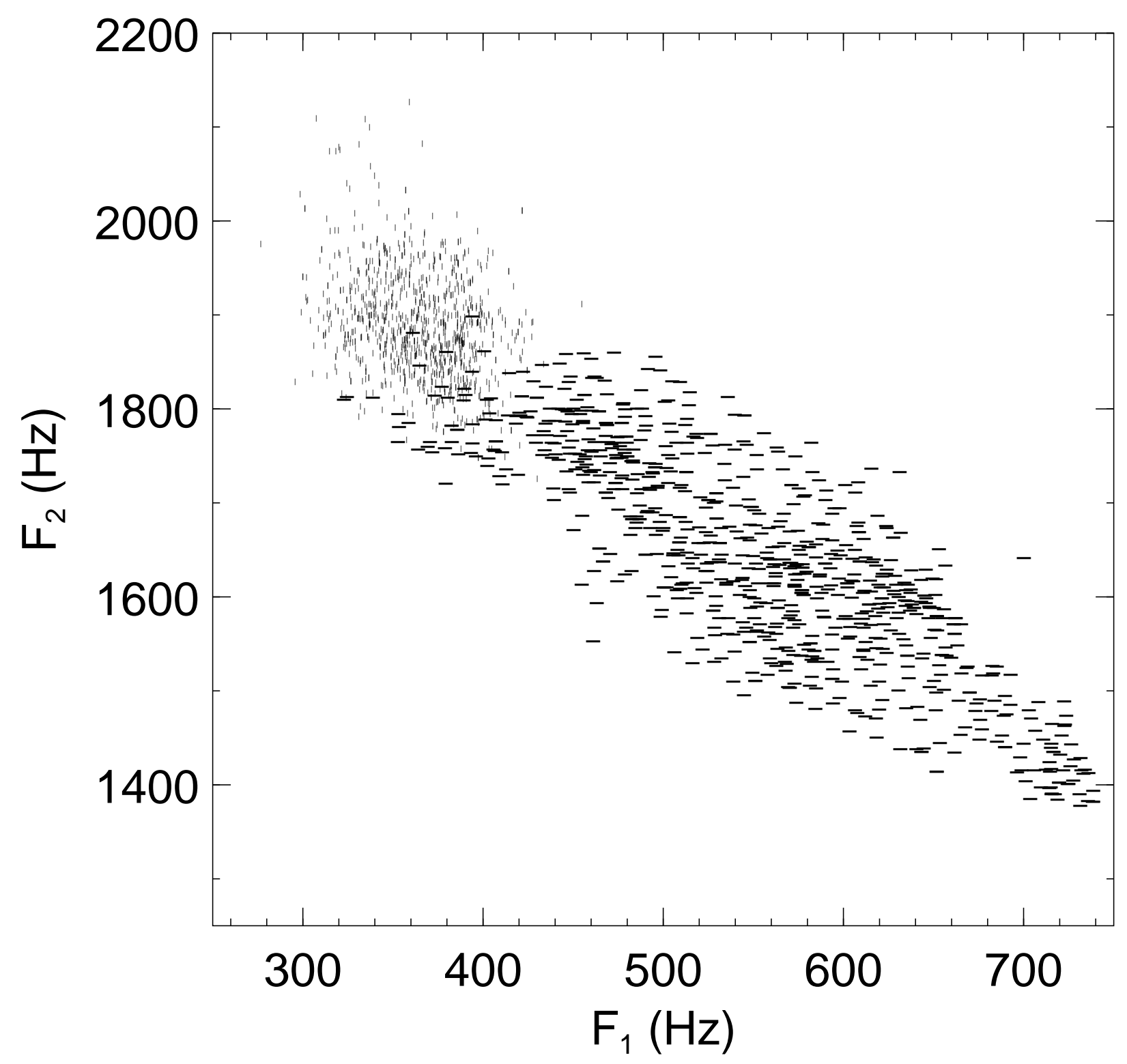


FIG. 9. Michel Pitermann, The Journal of the Acoustical Society of America.

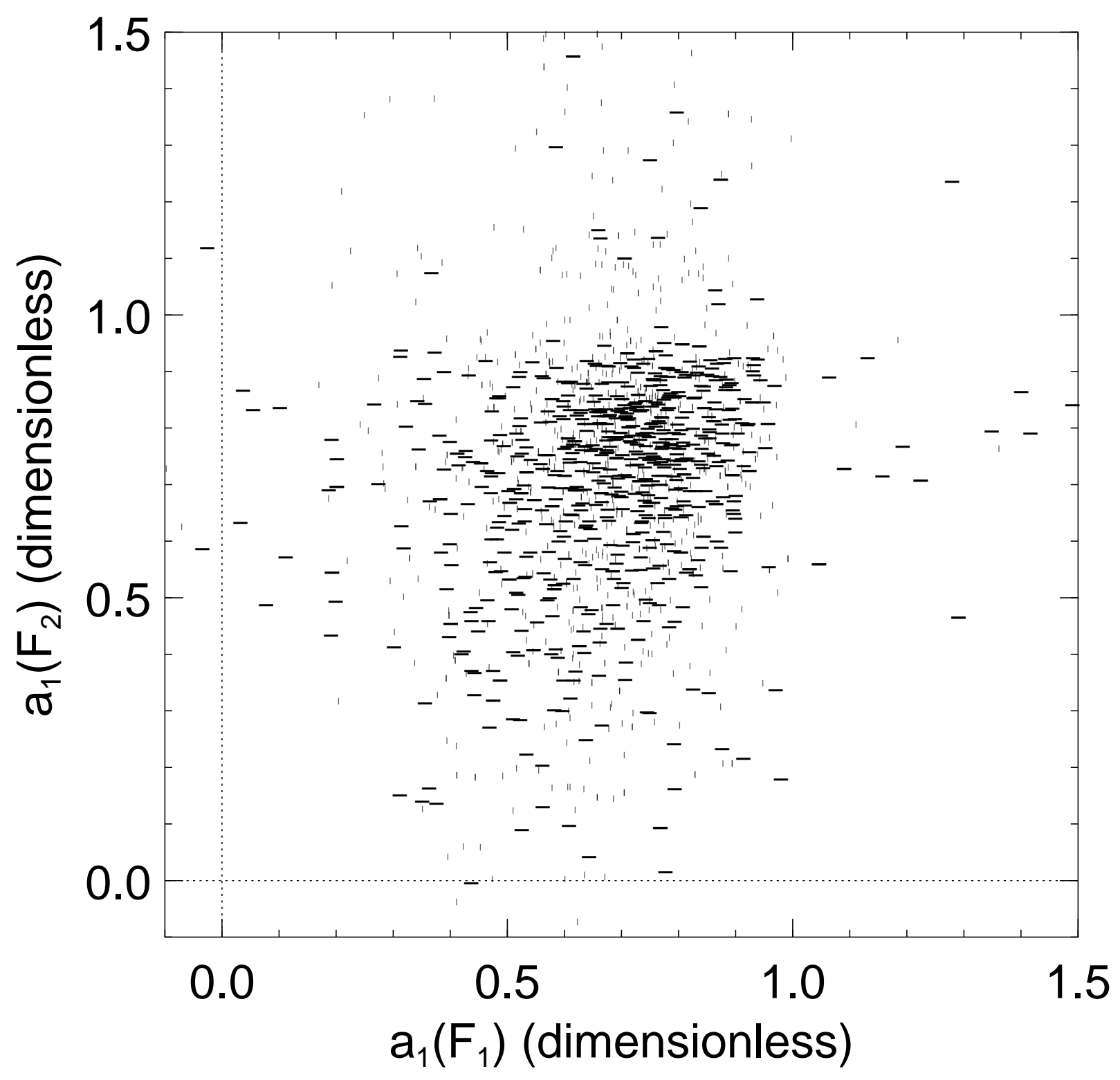

